\title{
Low-Mass Eclipsing Binaries to Refine Barnes-Evans-Like Relations
}

\author{
Pedro J. Amado \\ Dpto. de Física Teórica y del Cosmos, Facultad de Ciencias, Universidad de Granada, Spain; \\ Instituto de Astrofsica de Andalucía-CSIC, Granada, Spain \\ email: pja@iaa.es
}

\begin{abstract}
.
The relation of the surface brightness, a parameter related to the apparent magnitude and the angular diameter of a star, with a colour index is used to determine various stellar parameters. Eclipsing spectroscopic binaries can even be used as a distance indicator. In this work, we use a number of low-mass eclipsing binaries, whose parameters have been accurately determined, to refine the Barnes-Evans-like relation $F_{V}-\left(I_{\mathrm{c}}-K\right)$ introduced by Amado et al. (1999), a relation more suitable for the low temperature of these cool stars.
\end{abstract}

Keywords. binaries: eclipsing; stars: fundamental parameters; stars: late-type; stars: low-mass

\section{Introduction}

Calibration of surface brightness against colour index has a very wide range of applicability, amongst which is the evaluation of various stellar parameters. Wesselink (1969) and later Barnes \& Evans (1976) calibrated this parameter against various colour indexes and discussed their usefulness. Since then, many other authors have undertaken such calibrations against optical and infrared colour indexes with improved and more accurate data obtained by means of Michelson interferometry or by using model atmospheres (Barnes et al. 1978; Bell \& Gustafsson 1980; Moon 1984).

Table 1. Eclipsing binaries used in this study

\begin{tabular}{lccl}
\hline Name & $\left(I_{\mathrm{c}}-K\right)$ & $F_{V}$ & Ref. \\
\hline Barnard's Star & 2.260 & 3.269 & Dawson \& De Robertis (2004) \\
CU Cnc & 2.464 & 3.246 & Ribas (2003) \\
& & 3.198 & \\
V818 Tau & 0.790 & 3.749 & Torres \& Ribas (2002) \\
& 1.226 & 3.555 & \\
GU Boo & 1.578 & 3.465 & López-Morales \& Ribas (2005) \\
& 1.867 & & \\
CM Dra & 2.210 & 3.250 & Viti et al. (1997) \\
& & 3.271 & \\
YY Gem & 1.971 & 3.386 & Torres \& Ribas (2002) \\
Sun & 0.850 & 3.753 & Allen (1973) \\
TrES-Her0-07621 & $?$ & $?$ & Creevey et al. (2005) \\
UNSW-TR-2 & $?$ & $?$ & Young et al. (2006) \\
OGLE BW3 V38 & $?$ & $?$ & Maceroni \& Montalbán (2004) \\
\hline
\end{tabular}




\section{The $F_{V}-\left(I_{\mathrm{c}}-K\right)$ relation}

Figure 1 shows the $F_{V}-\left(I_{\mathrm{c}}-K\right)$ relation determined by Amado et al. (1999) with the dwarfs (solid symbols) and giants (open symbols) data. A best bilinear fit was given in the paper and is shown as lines for the giants. Larger symbols are for binaries.

Data for dwarf stars were obtained from the literature (see Amado et al. 1999 and references therein) Values for eclipsing binaries were also compiled from the literature (see Table 1 for references). The linear fit:

$$
F_{\mathrm{V}}=3.997-0.311\left(I_{\mathrm{c}}-K\right)
$$

is shown in Figure 2 together with the dwarf and binary data.

\section{Discussion}

The dispersion seen in the data around the fits may be due to:

(a) intrinsic variability

(b) Non-simultaneous $I$ and $K$ magnitude measurements

(c) Effect of metallicity (Beuermann et al. 1999)

(d) $V$ and $\left(I_{\mathrm{c}}-K\right)$ not corrected for interstellar reddening

The radii of dwarf stars determined from the use of PHOENIX model atmospheres (Leggett 1996) and used to compute $F_{V}$ agree with those determined empirically for eclipsing binaries.

New determinations of the parameters of YY Gem (Torres \& Ribas 2002) place the star on the locus defined by the rest of the dwarfs and binary stars.

\section{Conclusions}

The use of this relation, optimized for cool main-sequence stars, together with that for giants (Amado et al. 1999) provides a means to determine parameters such as effective temperatures and radii for stars with measured colours and distances. For eclipsing binaries with good determination of the radii of the components the relation provides the distance to the system.

\section{Future work}

Simultaneous out-of-eclipse photometric observations and calibrations to the $I$ and $V$ band are needed for the individual components of the binaries to produce more accurate $\left(I_{\mathrm{c}}-K\right)$ colours.

\section{Acknowledgements}

The author acknowledges financial support from a "Ramón y Cajal" contract (RYC2005-000208). 


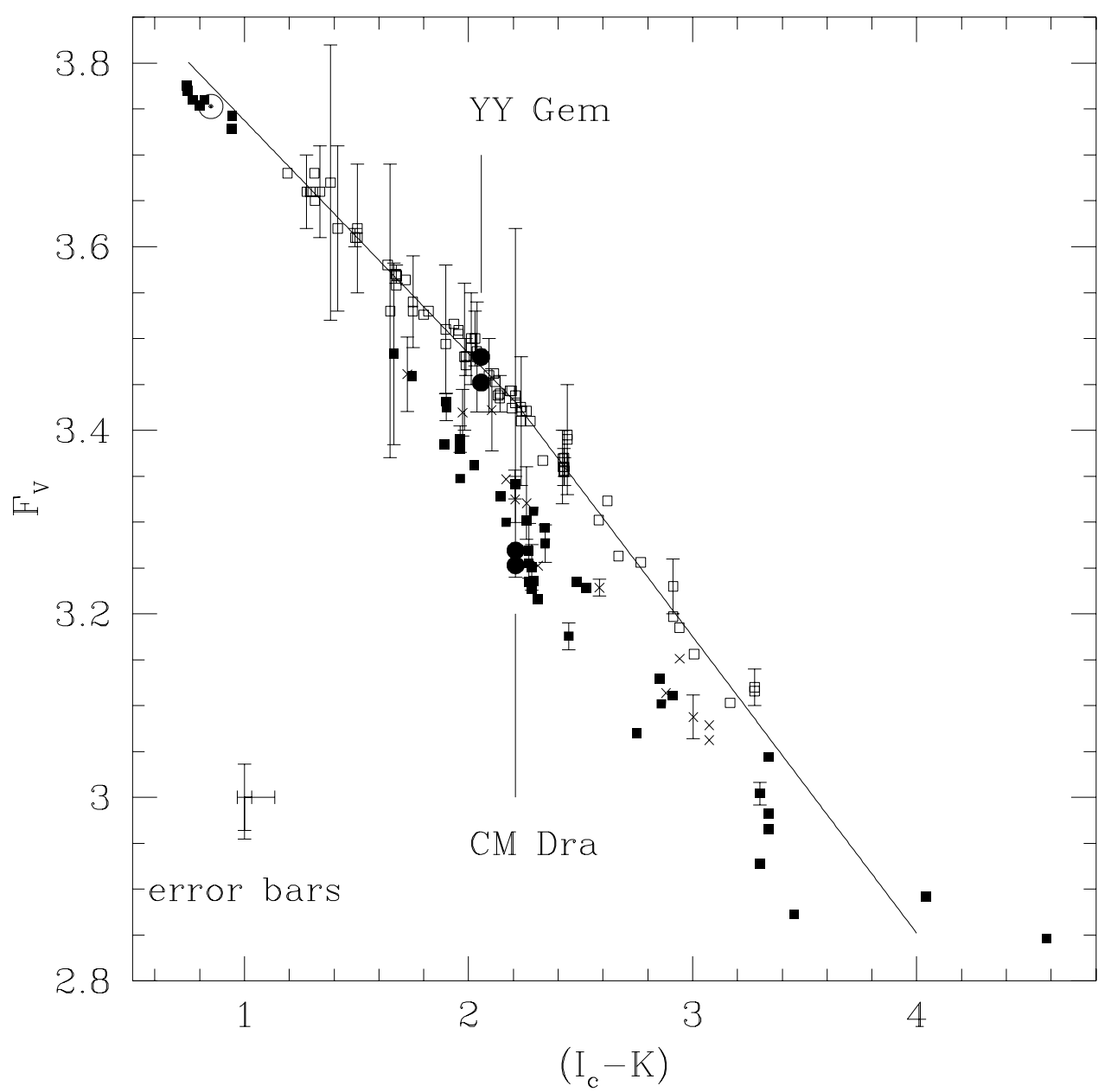

Figure 1. Calibration of the surface brightness parameter $F_{V}$ against the $\left(I_{\mathrm{c}}-K\right)$ colour index. The solid line represents the best bilinear fit to the giant stars (open square symbols). Filled squares and crosses are dwarf single stars and unresolved binaries respectively, with radii determined from model atmospheres or blackbody curves. The Sun is represented by $\odot$ and YY Gem and CM Dra by filled circles (radii determined from eclipses). The error bars shown by the smaller symmetrical cross represent a maximum for the errors introduced in the $F_{V}$ determination and in the $\left(I_{\mathrm{c}}-K\right)$ colour of YY Gem and CM Dra by the errors in the radii and distance and the standard errors in the photometry. The errors given by the larger asymmetrical cross represent an estimate of the indeterminacy introduced in $F_{V} \mathrm{q}$ and the colour by starspots on these two stars.

\section{References}

Allen, C.W. 1973, Astrophysical Quantities, Athone Press, London Amado, P.J., Butler, C.J., \& Byrne, P.B. 1999, MNRAS, 310, 1023

Barnes, T.G. \& Evans, D.S. 1976, MNRAS, 174, 489

Barnes, T.G., Evans, D.S., \& Moffett, T.J. 1978, MNRAS, 183, 285

Bell, R.A. \& Gustafsson, B. 1980, MNRAS, 191, 435 


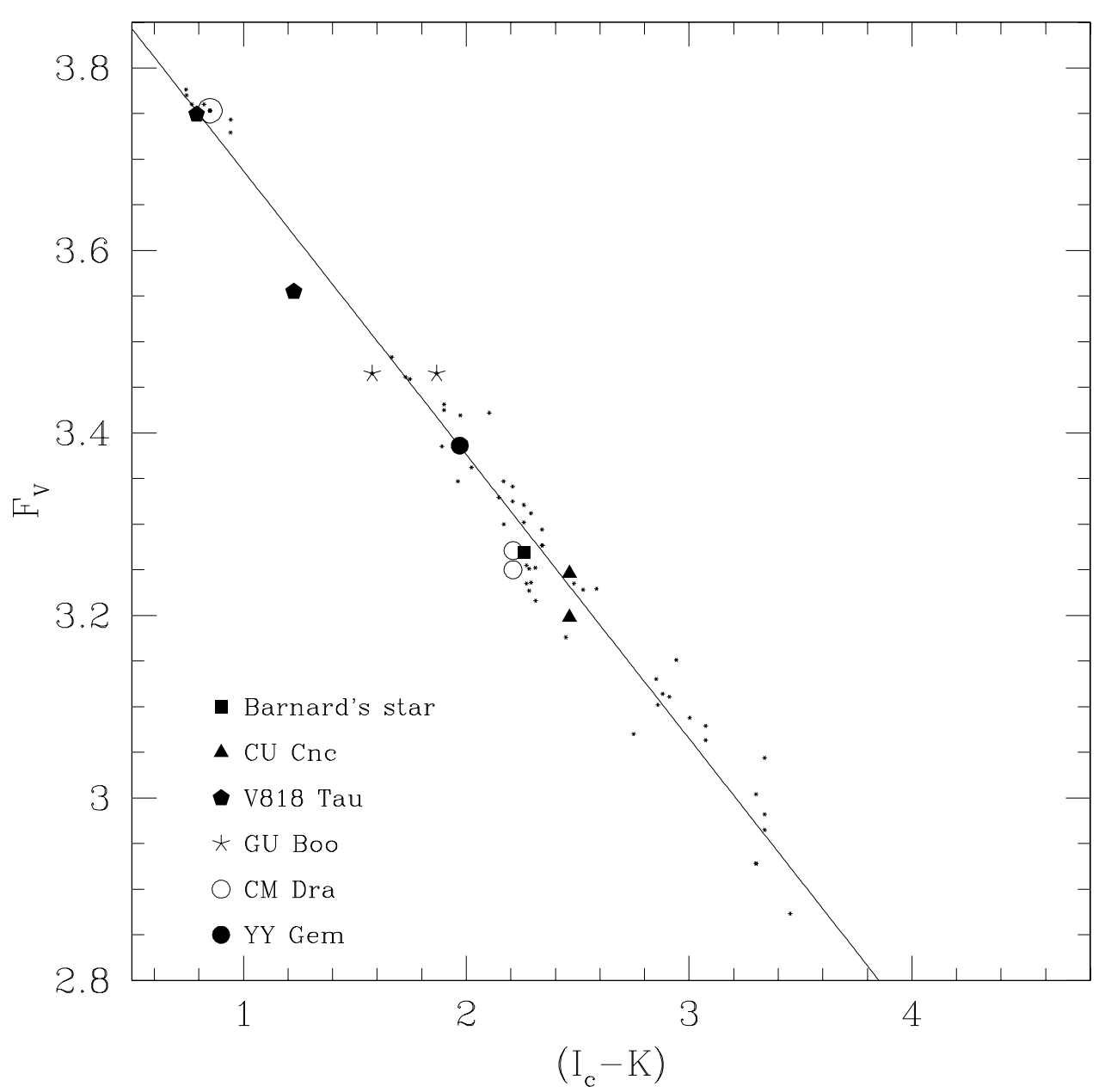

Figure 2. Data for dwarf stars (small dots) and eclipsing binaries (larger symbols) together with the best fit.

Beuermann, K., Baraffe, I., \& Hauschildt, P. 1999, A\& A, 348, 524

Creevey, O.L., Benedict, G.F., Brown, T.M., Alonso, R., Cargile, P., Mandushev, G.,

Charbonneau, D., McArthur, B.E., Cochran, W., O’Donovan, F.T., Jiménez-Reyes, S.J., Belmonte, J.A., \& Kolinski, D. 2005, ApJ, 625, L127

Dawson, P.C. \& De Robertis, M.M. 2004, AJ, 127, 2909

Leggett, S.K., Allard, F., Berriman, G., Dahn, C.C., \& Hauschildt, P.H. 1996, ApJS, 104, 117

López-Morales, M. \& Ribas, I. 2005, ApJ, 631, 1120

Maceroni, C. \& Montalbán, J. 2004, A\&广A, 426, 577

Moon, T.T. $1984, M N R A S, 211,21$

Ribas, I. 2003, $A \mathscr{E} A, 398,239$

Torres, G. \& Ribas, I. 2002, ApJ, 567, 1140

Viti, S., Jones, H. R.A., Schweitzer, A., Allard, F., Hauschildt, P.H., Tennyson J., Miller, S., \& Longmore, A.J. 1997, MNRAS, 291, 780

Wesselink, A.J. 1969, MNRAS, 144, 297

Young, T.B., Hidas, M.G., Webb, J.K., Ashley, M.C.B., Christiansen, J.L., Derekas, A., \& Nutto, C. 2006, MNRAS, 370, 1529 